\title{
Zum 100-Jahr-Jubiläum der Siegfriedkarte der Schweiz
}

Armin Bleuer

Am 18. Dezember 1868 wurden zwei Bundesgesetze erlassen, die für die Entwicklung des schweizerischen Kartenwesens von größter Bedeutung waren. Das eine betraf die Fortsetzung und das andere die Publikation der topographischen Aufnahmen. Die beiden Gesetze schufen die Grundlage für die Erstellung unserer Siegfriedkarte; da nun die eigentliche Geburtsstunde dieses Kartenwerkes genau 100 Jahre zurückliegt, scheint uns ein Rückblick gerechtfertigt.

General Dufour hatte seine berühmte Topographische Karte der Schweiz 1:100000 1864 zum Abschluß gebracht. Die Terrainaufnahmen erfolgten mit Höhenkurven, und zwar fürs Gebirge im Maßstab 1:50 000 und mit $30 \mathrm{~m}$ Äquidistanz und fürs Mittelland, den Jura und das Südtessin im Maßstab 1: 25000 und mit einer Äquidistanz von $10 \mathrm{~m}$. Da von größeren Teilen des Landes bereits Schraffenkarten bestanden, wurden diese, sofern sie nach maßstäblicher Verkleinerung als brauchbar erachtet wurden, als Grundlage verwendet. Es handelte sich um die bestehenden Karten folgender Kantone: Appenzell A.- und I.-Rh. (von Mertz), Thurgau (von Sulzberger), Aargau (von Michaelis), Basel-Stadt und -Land (von Baader), Solothurn (von Walker), Berner Jura (von Buchwalder) und Neuenburg (von Osterwald).

Auf Ende 1864 trat General Dufour zurück und empfahl als Nachfolger den damaligen Major im Generalstab Siegfried, der denn auch im folgenden Jahr die Leitung des Eidg.Topographischen Bureaus übernahm und zum Oberstleutnant befördert wurde. Gleichzeitig wurde das Bureau von Genf nach Bern verlegt. Die Aufgaben waren vorest bescheiden und umfaßten zur Hauptsache die Fortsetzung des Stiches der "Reduzierten Karte» oder vierblättrigen Generalkarte 1:250 000 und die Vervollständigung des Atlanten 1: 100000 durch Nachführen der seit der Zeit der Aufnahmen erfolgten Nachträge und durch Verbesserungen festgestellter Mängel.

In den 60er Jahren des vorigen Jahrhunderts wurde das wirtschaftliche Bild der Schweiz durch den überall stark forcierten Eisenbahnbau und die stark aufkommende Industrialisierung geprägt. Es ist daher nicht verwunderlich, wenn an die Kartierung des Landes bald höhere Ansprüche gestellt wurden, als sie eine noch so schöne Darstellung in Schraffenmanier $1: 100000 \mathrm{zu}$ befriedigen vermochte. Es waren wohl einige Karten in größerem Maßstab nach den Aufnahmen, die Dufour als Grundlage dienten, hergestellt worden. Es seien hier bloß die von 1849-1852 geschaffene Schraffenkarte $1: 25000$ des Kantons St. Gallen und die hervorragende Wild-Karte 1:25000 des Kantons Zürich erwähnt. Letztere entstand von 1852-1865, wurde als Kurvenkarte in vier Farben auf Stein graviert und darf noch heute als beispielhaft angesprochen werden.

Diese kantonalen Erzeugnisse boten aber ein im ganzen sehr uneinheitliches und lückenhaftes Bild, so daß das Fehlen einer größeren Gesamtkartierung der ganzen Schweiz immer mehr empfunden wurde. Die 1859 gebildete Geologische Kommission der Schweizerischen Naturforschenden Gesellschaft richtete daher 1862 durch ihre Mitglieder Alphonse Favre in Genf und Bernhard Studer in Bern ein Gesuch an den Bundesrat, die Eidgenossenschaft möge eine Karte 1:50 000 mit Höhenkurven von $30 \mathrm{~m}$ Äquidistanz herausgeben. Das Gesuch wurde aber abgelehnt mit der Begründung, da $\beta$ andere bedeutende Ausgaben dem Bund in nächster Zeit genug zu schaffen gäben.

Der 1863 gegründete Schweizer Alpenklub setzte sich von allem Anfang an für die Publikation guter Tourenkarten ein; schon den Jahrbüchern 1863 und 1865 konnte er die mit Bewilligung des Eidg.Topographischen Bureaus hergestellten Klubkarten vom Tödi-Clariden- und Triftgebiet beigeben. Die dritte Generalversammlung 1865 in Chur faßte folgenden Beschluß: "Das Zentralkomitee ist beauftragt, im Einverständnis mit andern Vereinen, die ähnliche Zwecke verfolgen wie der SAC, dahin zu wirken, daß die eidgenössische topographische Karte im Maßstab und in der Manier der Originalaufnahmen veröffentlicht werde.» Dieser Beschluß fand aber bei den anvisierten Vereinen und Gesellschaften keine Unterstützung. Trotzdem bemühte sich der SAC in den folgenden Jahren weiterhin intensiv. um die Kartenherstellung. Er beauftragte besonders J. Coaz, den ehemaligen Ingenieur des topographischen Bureaus und Oberforstinspektor, mit der Angelegenheit. $\mathrm{Zu}$ der beabsichtigten Eingabe an die Bundesbehörden kam es aber offenbar nie. Es darf freilich angenommen werden, daß die Anliegen des SAC bei Dufour (Ehrenmitglied) und Siegfried (Mitglied) auf großes Wohlwollen stießen und daß hier in persönlichem Kontakt manche Auffassung geklärt wurde. 
Gesuche um Benützung der Originalaufnahmen $1: 25000$ und $1: 50000$ und deren Publikation durch verschiedenste Interessenten häuften sich immer mehr, so daß sich ein grundsätzlicher Entscheid aufdrängte. Am 16. November 1867 begründete Siegfried die Ablehnung eines weiteren Gesuches für die Publikation der Originalaufnahmen gegenüber dem Chef des EMD wie folgt: "Mit meinem Antrag, keine weiteren Kopien zu gestatten, muß ich den Vorschlag verbinden, die Publikation der Originalaufnahmen solle von der Eidgenossenschaft selber an die Hand genommen werden.» Große Summen lägen in den Aufnahmen, durch die wilde Publikation von Privaten führe es aber zum Chaos, zur Uneinheitlichkeit in Formaten und Maßstäben. Der Publikation müsse ein einheitlicher Plan zugrunde liegen. Gleichförmigkeit in der Ausführung erhalte man nur durch sachverständige Aufsicht. Jede Buchhandlung könne für ein Stück, das gerade Absatz verspreche, die Bewilligung verlangen und es publizieren. Eine solche Ausbeutung dürfe die Behörde nicht gestatten. Gleichzeitig stellte er den Antrag, das Stabsbüro sei mit der Vorlage eines Planes zur sukzessiven Publikation der Aufnahmeblätter zu beauftragen.

Schon am 11. Januar 1868 konnte Siegfried einen solchen Plan einreichen. Er schlug dabei vor, das Format einheitlich gleich wie dasjenige der Aufnahmeblätter, d. h. $26 \times 35 \mathrm{~cm}$, zu wählen. Es sei wohl klein, aber gerade dadurch sei es günstiger, die Blätter je nach Wunsch zusammensetzen zu können. Der Chef des EMD ließ diesen Plan durch eine Kommission überprüfen, die aus Oberst und Ingenieur L. S. Delarageaz aus Lausanne, Präsident, Ingenieur und Professor Joh. Wild aus Zürich und Forstinspektor J. Coaz aus Chur bestand. Oberst Siegfried nahm an den Verhandlungen ebenfalls teil.

Die Anträge dieser Kommission deckten sich im allgemeinen mit den Vorschlägen Siegfrieds. Sie waren erfolgreich und führten schließlich zum Erlaß der beiden eingangs erwähnten Bundesgesetze. Diese sahen vor, daß für alle Gebiete der Dufourkarte, für die nur kantonale Schraffenkarten als Grundlagen dienten, topographische Aufnahmen $1: 25000$ mit Höhenkurven von $10 \mathrm{~m}$ auszuführen seien, deren Kosten von Bund und Kantonen zu gleichen Teilen getragen werden mußten. Der Publikation jedes Kartenblattes hatte eine Revision, Ergänzung oder Umarbeitung der Aufnahmen vorauszugehen. Für den Gesamtatlas waren nach dem aufgestellten Plan vorgesehen: 429 in Kupfer gestochene Blätter 1 : 25000 für den Jura, das Mittelland und das Südtessin und 117 durch Steingravur erstellte Blätter 1 : 50000 der vor- und hochalpinen Gegenden der Schweiz. Für die Durchführung der gesamten Publikation wurde mit 25 bis 30 Jahren gerechnet.

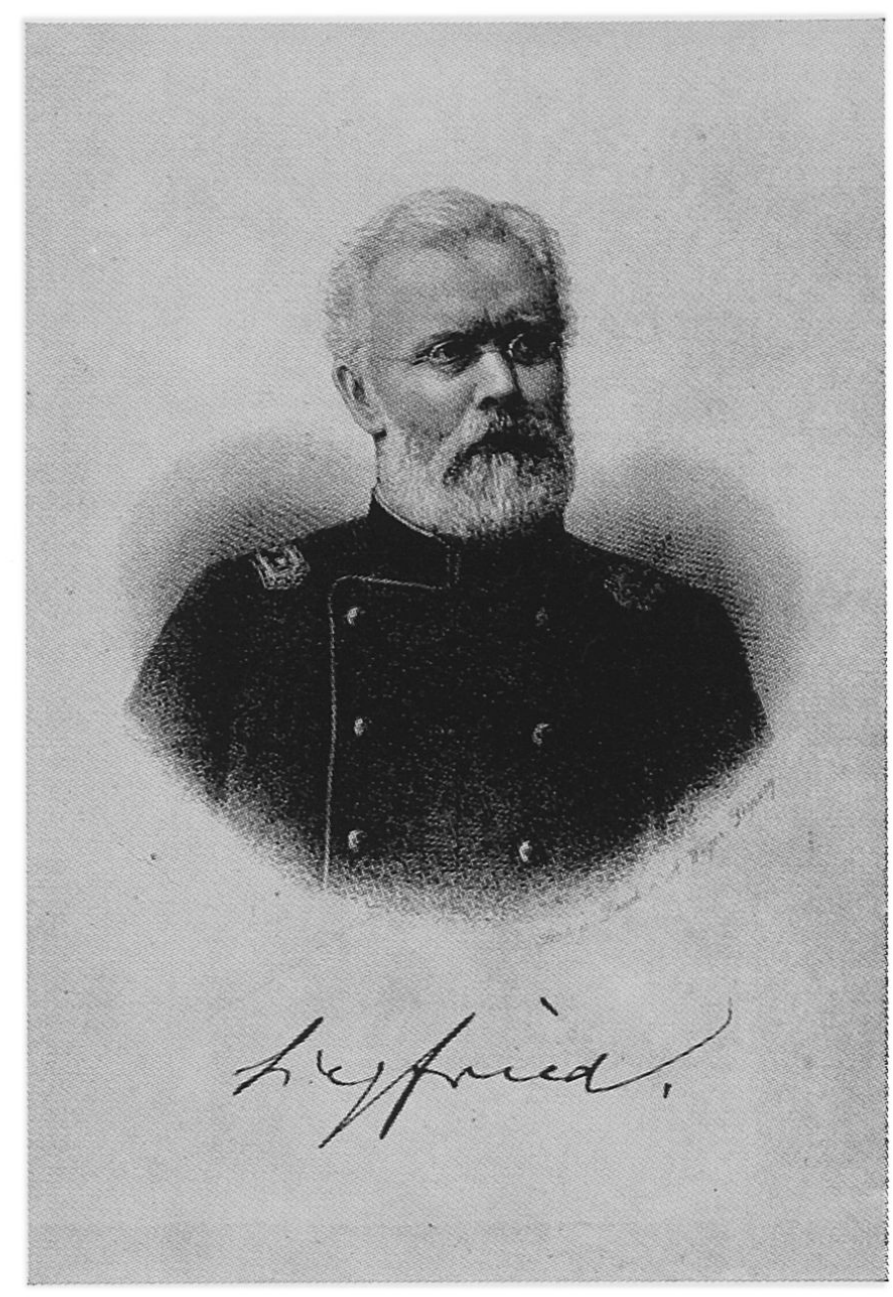

Abbildung 1. Oberst Hermann Siegfried

(19. Februar 1819 bis 5. Dezember 1879), Schöpfer des nach ihm benannten Topographischen Atlas der Schweiz

Die topographischen Aufnahmen und Revisionen für die zur Publikation gelangenden Aufnahmeblätter wurden von den Ingenieuren des Eidg. Topographischen Bureaus ausgeführt nach Instruktionen, die Oberst Siegfried auf Grund seiner langen Erfahrung als Topograph im Dienste Dufours aufgestellt hatte. Die Publikation erfolgte in sogenannten Lieferungen zu 12 bis 14 Blättern. Die erste erschien bereits Ende 1870, und die weitern folgten sich in regelmäßigen Abständen bis zum Jahre 1901, als mit der 49. Lieferung die Publikation mit 581 Blättern praktisch zum Abschluß kam. Von diesem Zeitpunkt an erschienen noch vereinzelte Neuausgaben, so daß der gesamte Siegfriedatlas schließlich 604 Kartenblätter umfaßte, davon 462 im Maßstab $1: 25000$ und $142 \mathrm{im} \mathrm{Maßstab} 1: 50000$.

Bis 1949 wurde am ganzen Kartenwerk weitergearbeitet. Jedes Blatt kam durchschnittlich 4- bis 5mal zur Nachführung, und über 20 Blätter, die nicht ganz befriedigten, wurden nochmals neu aufgenommen oder doch wenigstens vollständig neu bearbeitet. 


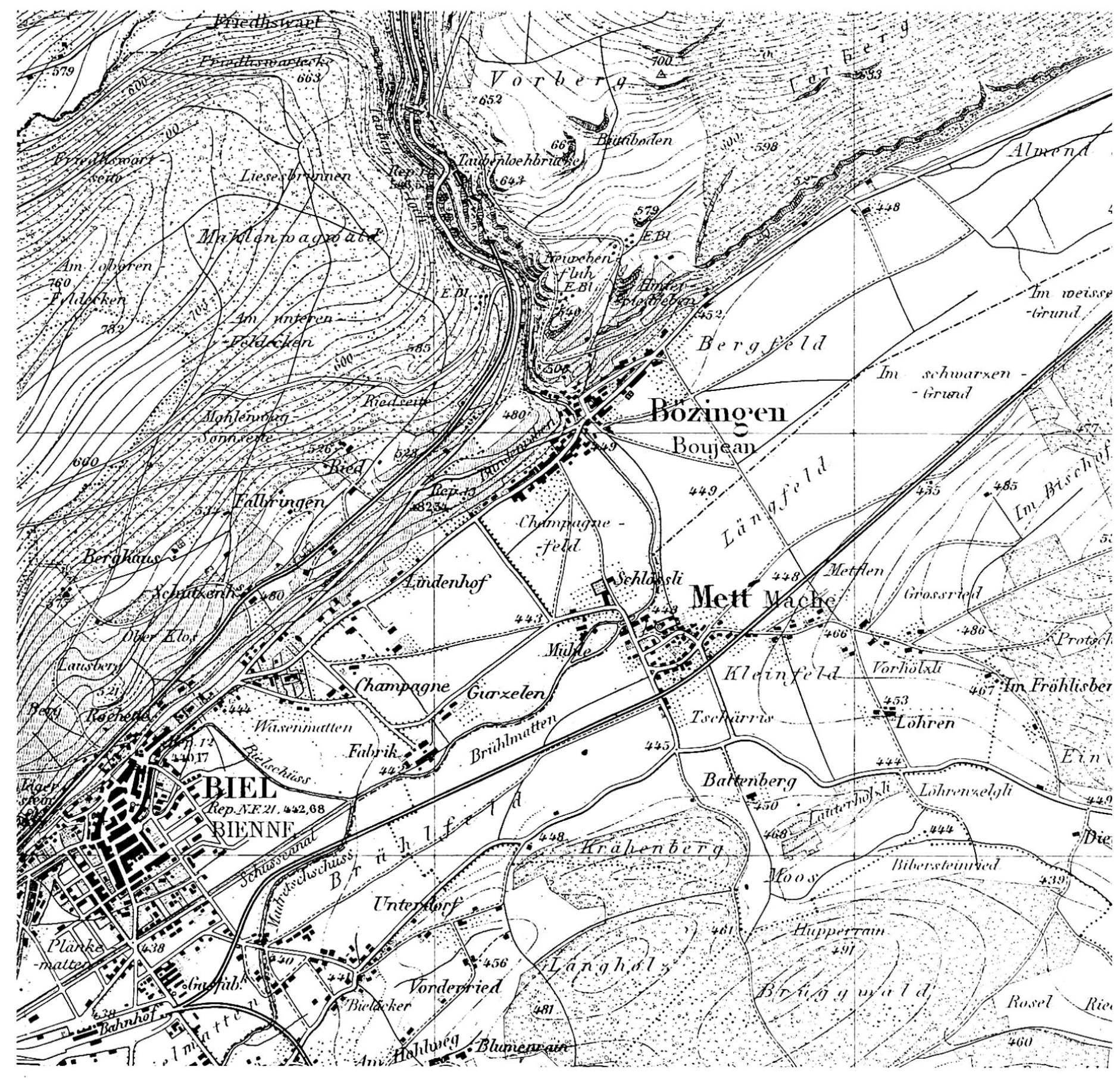

Ábbildung 2. Ausschnitt aus Blatt 124 «Biel» der Siegfriedkarte, Erstausgabe aus dem Jahre 1876. Die gewaltigen seither eingetretenen kulturlandschaftlichen, namentlich städtebaulichen Veränderungen sind beim Vergleich mit dem heutigen Zustand augenfällig. (Wiedergabe mit Bewilligung der Eidg. Landestopographie.)

Oberst Siegfried selbst hat sich kurz vor seinem Tode im Jahre 1879 über die Entstehung seines Werkes wie folgt geäußert: «In den meisten Staaten, welche die Aufnahmen im Originalmaßstab publizieren, wird auf die Schönheit der Ausgabe wenig gesehen, sondern das Gewicht darauf gelegt, in kürzester Zeit nach vollendeter Aufnahme und mit den geringsten Kosten dem Publikum die neuen Blätter liefern zu können. In der Schweiz hat man andere Ansprüche an diese Publikation gestellt. Die Ausgabe wird mit aller Sorgfalt als ein Hauptwerk unserer Topographen und nicht bloß als ein provisorisches Hilfsmittel für spezielle Zwecke behandelt. Es ist dies denjenigen vielleicht nicht recht, welchen die Publikation nicht rasch genug erfolgen kann. Das Publikum hingegen ist mit dieser Ausführung zufrieden und freut sich der Anerkennung, welche dieselbe auch im Auslande bei Fachmännern gefunden hat.» In dieser Äußerung kommt die Grundhaltung zum Ausdruck, die unsere Vorfahren beseelte und die den hervorragenden Ruf der schweizerischen Kartographie begründete. 
Die Siegfriedkarte brachte zum ersten Mal die Topographie und die Besiedlung unseres Landes in großem Detailreichtum zur Darstellung. Einzelne Blätter dürfen als wahre Kunstwerke gelten, ist es doch den Topographen und Kartolithographen gelungen, durch eine raffinierte Felszeichnung unser Gebirge höchst anschaulich wiederzugeben. Es ist daher nicht verwunderlich, daß dieses Kartenwerk überall Anerkennung gefunden hat und auf den verschiedensten Gebieten menschlicher Betätigung wertvollste Dienste leistete. Während zweier Weltkriege gehörte der Atlas zudem zur Ausrüstung unserer Armee.

Vor Jahren war es verschiedentlich Mode geworden, die Güte der Siegfriedkarte anzuzweifeln und das Mißlingen von Artillerieschießen wollte man vielfach auf ihr Ungenügen zurückführen, dabei lag die Ursache doch meistens im unrichtigen Lesen der Karte. Wenn wir zu einem gerechten Urteil kommen wollen, müssen wir uns vergegenwärtigen, daß die meisten Aufnahmen ja nur zum Zwecke der Erstellung der Dufourkarte 1:100000 erfolgt waren. Wohl wurden diese Aufnahmen, wie bereits erwähnt, vor der Publikation revidiert und Teile davon überhaupt neu erstellt. Es konnte aber dadurch kein Werk entstehen, das heutigen Genauigkeitsanforderungen genügen konnte. Trotz allem Bemühen wiesen die Blätter qualitative Unterschiede auf. Neben solchen von verblüffender Genauigkeit gab es auch vereinzelte Hochgebirgsblätter mit Lagefehlern von einigen hundert Metern. Es wäre aber höchst ungerecht, durch solche Einzelfälle das hohe Werturteil über die Siegfriedkarte stark zu beeinträchtigen.

Schon recht früh wurde als Nachteil empfunden, daß sich das Kartenwerk aus zwei Maßstäben zusammensetzte, daß also längs den Voralpen ein Sprung von $1: 25000$ in $1: 50000$ bestand. Bereits 1890 setzten Bestrebungen ein mit dem Ziel der Erstellung einer Kurvenkarte 1:50000 mit plastischer Terraindarstellung der ganzen Schweiz. Um die Jahrhundertwende trat besonders von militärischer Seite das Verlangen nach einer mehrfarbigen Kurvenkarte 1:100000 als Ersatz des Dufouratlas in den Vordergrund. Die Eidg. Landestopographie wurde mit entsprechenden Vorarbeiten betraut, und dabei zeigte sich, daß eine Großzahl von Siegfriedblättern den gestellten hohen Anforderungen nicht entsprach und daß für mehr als die Hälfte der Blätter 1: 25000 und fünf Sechstel aller Blätter 1:50 000 entweder weitläufige Revisionen oder sogar Neuaufnahmen vorzusehen waren. Zum Glück verhinderte der Ausbruch des 1. Weltkrieges die Inangriffnahme dieser Arbeiten, denn die moderne Aufnahmemethode der Photogrammetrie, die heute mit verhältnismäßig kleinem Aufwand früher unerreichbare Genauigkeiten liefert, stand erst viel später zur Verfügung. Die erwähnten Wün- sche und Begehren nach neuen Kartenausführungen fanden erst 1935 im Bundesgesetz über die Erstellung der neuen Landeskarten ihre Berücksichtigung.

Der heutigen Generation ist die Siegfriedkarte hautpsächlich in der Ausgabeform von Zusammensetzungen bekannt. Zur Publikation gelangten deren 27 im Maßstab 1:50000 und 24 im Maßstab $1: 25000$. Jede dieser Zusammensetzungen bildet den Bereich mehrerer Einzelblätter ab. Diejenigen 1 : 50000 umfassen das gesamte vor- und hochalpine Gebiet, während die im Maßstab 1:25000 vorhandenen sich auf Gegenden im Bereiche der Waffenplätze, der größeren Städte und auf Exkursionsgebiete ausdehnen und zudem einen grünen Waldton aufweisen.

Ende der vierziger Jahre wurden die Nachführungsarbeiten für den Siegfriedatlas endgültig eingestellt. Nachdrucke sind heute nicht mehr möglich, und das ganze Kartenwerk hat daher nur noch historischen Wert. Es wird aber weiterhin eine für die damalige Zeit ganz außerordentliche Leistung bezeugen, und kein Fachmann wird je den Schöpfern seine Hochachtung versagen können.

\section{Quellen}

Schneider, Karl: Geschichtlicher Streifzug durch die ersten hundert Jahre Eidg. Landestopographie 1838 - 1938 .

Oberli, Alfred: Wie es zur Herausgabe der Siegfriedkarte kam.

\section{Résumé}

Deux lois fédérales ont été publiées le 18 décembre 1868: l'une concernait la poursuite des levés topographiques, alors que la seconde traitait de la publication de ces mêmes levés; elles constituaient les bases pour l'établissement de la carte Siegfried, dont quelque 600 feuilles ont été publiées de 1870 à 1901. Le Plateau suisse, le Jura et le Tessin méridional devaient être représentés à l'échelle 1:25000, avec courbes de niveau équidistances de 10 mètres, alors que le 1:50000 et une équidistance de 30 mètres étaient retenus pour les Alpes et les Préalpes. Les levés originaux dataient essentiellement de l'époque de Dufour.

La beauté et la grande exactitude de la plupart des feuilles de l'Atlas Siegfried ont été largement appréciées. Jusqu'à l'issue de la Seconde Guerre mondiale, cette œuvre cartographique a connu d'innombrables emplois; le centième anniversaire de son lancement méritait donc qu'on en retraçât le cours. La carte Siegfried est actuellement remplacée par les nouvelles cartes nationales et n'a plus de valeur qu'historique. 